\section{Response to both}

When developing our review we were keen to generate discussion with a view to raising awareness of, and improving practice around, discharge planning. We therefore appreciate the comments of Drs Levine and Leung.

We agree that sharing accurate information in a timely manner is a key factor in both discharge planning and provision of high-quality care. The presence on ward rounds of nursing staff should improve this, but can be suboptimal. Combined paperwork and single assessment pathways, alongside daily targeted multidisciplinary meetings have been used in the admission unit at Stafford with some effect. Many other assessment units (eg Wolverhampton) provide active elderly care in-reach services and/or regular consultant input, potentially facilitating more appropriate discharge and admission.

Admissions units are a hub in most acute hospitals, and accurate assessment and decision making, with early senior clinician involvement at the point of admission, can ultimately improve care downstream. We would endorse the points raised by Dr Leung regarding the potential conflict between early discharge and the provision of high-quality care, and the fact that many frail patients with complex medical conditions and social circumstances cannot be discharged directly from admissions units.

Pathways/protocols for early discharge of patients with selected conditions (eg deep vein thrombosis, cellulitis) from admission units have been described. ${ }^{1,2}$ We also recognise concerns of colleagues around setting discharge dates, and use the term 'provisional discharge date' in the notes, thus allowing flexibility.

Comprehensive assessment for many patients requires admission to specialist elderly care wards, where time will allow multidisciplinary assessment to occur. Our review aims to improve discharge planning both from admissions areas and specialist wards, improving the flow of patients through hospital, to allow more efficient use of resources.
Regular formal multidisciplinary meetings that document clear plans, proposed timescales, and individual responsibilities (either in clinical notes or on multidisciplinary handover sheets) can advance this process. We have also found that whiteboards are helpful in focusing actions of members of the multidisciplinary team.

Discharging patients, both from admissions unit and elderly care wards, can be a challenging process, and should be actively taught to doctors in training. Unsafe discharges reflect poor care and are unacceptable.

PHILIP DAINTY

Locum consultant physician Emergency Assessment Unit New Cross Hospital, Wolverhampton JIMMY ELIZABETH

Consultant physician Stafford General Hospital, Stafford

\section{References}

1 Boccalon $\mathrm{H}$, Elias A, Chale JJ et al. Clinical outcome and cost of hospital vs home treatment of proximal deep vein thrombosis with a low-molecular-weight heparin. Arch Intern Med 2000;160: 1769-73.

2 Corwin P, Toop L, McGeoch G et al. Randomised controlled trial of intravenous antibiotic treatment for cellulitis at home compared with hospital. BMJ 2005; 330:29-135.

\section{A postal survey of doctor's attitudes to becoming mentally ill}

Editor - I read with great interest Hassan et als excellent paper (Clin Med August 2009 pp 327-32). There is much research and publicity, for example a recent Department of Health review, ${ }^{1}$ which finds that there are higher rates of psychological illnesses in doctors than the general population, so it is surprising to find in this study that the majority of doctors, especially psychiatrists, were not aware of this fact. Overall it seems to me that as a profession we are having the same 'conversation' about this issue. The debate on how to provide services for sick doctors has continued in a similar way for years. One of the factors that bothers doctorpatients most, indeed many patients, is the stigma of illness and sickness absence, as is borne out in this study. It is interesting that in reality most doctors do not lose their jobs, or have any long-term problem resulting from the fact of a particular diagnosis or sickness absence. It is true that long-term sickness absence requires a multidisciplinary approach and that the earlier a problem is dealt with the better. Many of a doctor's problems with illness arise from a denial of them. Confidentiality, which is different, and as borne out in this study, is the key to managing these situations. Many services for doctors do exist, can be accessed via their general practitioners or occupational health departments, and can be off-site from the workplaces.

I am surprised that most respondents say they would talk to family and friends because in many cases, in my experience as an occupational physician, once doctors and other people are ill, they find that admitting illness to family is difficult. Those answers smack of denial as well and may be a source of information bias in the study.

Most interesting is that the authors say that these attitudes and perceptions about doctors' health should be tackled and changed at medical school level as I am currently carrying out this kind of work with students. I have found, sadly, that the attitudes written about in this paper are well-entrenched by mid-third-year stage.

CLARE RAYNER

Specialist occupational physician Trainer for National Education Programme

for Health and Work

Royal College of General Practitioners

\section{Reference}

1 Department of Health. Mental health and ill health in doctors. London: DH, 2008.

\section{The skin in general medicine}

Editor - Dhoat and Rustin reviewed an important but often neglected part of general medicine in their article (Clin Med 
August 2009 pp379-84). We would like to add that xanthomatoses, especially xanthelasmas, are also an important marker for diabetes, propensity to coronary artery disease $(\mathrm{CAD})^{1}$ and gout ${ }^{2}$ apart from cholestasis and hyperlipidemia as mentioned. Besides xanthaelasma and xanthoma, presence of arcus juvenilis in young people (age $\leqslant 40$ years) may also be considered as a clinical sign for premature CAD. ${ }^{3}$ Premature graying and/or balding in chronic smokers has also been shown to be associated with premature CAD. ${ }^{4}$ Other cutaneous signs like ear lobe crease, ear canal hair, and nicotine staining should be considered as valuable clinical markers of CAD for the benefit of the readers. ${ }^{1}$ Recently, hyperpigmented palm and digits of hand associated with central obesity in betel quid sellers has been shown to predispose to early CAD. ${ }^{5}$ There may be a clinical scenario where one sibling in the family has xanthoma, other has xanthelasma or arcus juvenilis and some suffering from CAD. The clinical implication of such a finding is that one must actively look for such signs in all the family members for early identification of persons predisposed to premature CAD.

AMITESH AGGARWAL Lecturer

Department of Medicine/Preventive Cardiology University College of Medical Sciences University of Delhi, India

\section{References}

1 Chan HL. Cutaneous manifestations of cardiac diseases. Singapore Med J 1990;31:480-5.

2 Storey GO. Xanthomatosis, hyperlipoproteinaemia (type II, Fredrickson), gout, cardiac infarction. Proc R Soc Med 1971;64:59-60.

3 Chambless LE, Fuchs FD, Linn S et al. The association of corneal arcus with coronary heart disease and cardiovascular disease mortality in the Lipid Research Clinics Mortality Follow-up Study. Am J Public Health 1990;80:1200-4.

4 Dwivedi S, Aggarwal A. Risk factors and cutaneous markers of coronary artery disease in young in north Indian population. Atherosclerosis Supplement 2009; 10:e1406.

5 Dwivedi S, Dwivedi G. Betel quid seller syndrome. Occup Environ J 2009; forthcoming.

\section{Using umbilical cord blood stem cells for myocardial infarction and stroke is ethically challenging}

Editor - It is disingenuous for stem cell researchers such as Copeland et al (Clin Med August 2009 pp 342-5) to claim that ' $h U C B$ (human umbilical cord blood) is readily available, and presents little ethical challenges'. They must be aware that until the feto-placental circulation is interrupted by human professional intervention, that blood has a life-sustaining function. Without cord clamping, placental transfusion occurs and most of the blood then resides in the neonate. Pressure to separate mother and baby has created an apparent 'waste product' that may be valuable to researchers (and vested commercial interests). The earlier the cord is clamped, the more hUCB is 'readily available' for collection and the less is available for the neonate. Growing awareness of the impact of this irreversible intervention during the transitional circulation has led professionals responsible for the health and wellbeing of the new mother and baby to take a more cautious view. ${ }^{1}$ In view of findings of long-term developmental problems in children apparently successfully resuscitated after birth, we hypothesised that early clamping may lead both to neonatal depression and inadequate cerebral oxygenation. ${ }^{2,3}$ Physicians caring for adults and concerned about the alleviation of damage to adult vital organs must be aware of the ethical concerns about the use of vital material taken at birth.

SUSAN BEWLEY

Consultant obstetrician/maternal-fetal medicine Guy's \& St Thomas' NHS Foundation Trust, London

JUDITH MERCER

Clinical professor, University of Rhode Island, College of Nursing;

Adjunct professor, Department of Pediatrics, Brown University;

Research scientist, Women and Infants Hospital Rhode Island, USA

Conflict of interest: SB was a member of the Royal College of Obstetricians and Gynaecologists working party on umbilical cord stem cell collection. JM is PI of a research study into the protective effects of delayed cord clamping in very low birth weight infants.

\section{References}

1 Scientific Advisory Committee. Opinion paper 2. Umbilical cord blood banking. London: Royal College of Obstetricians and Gynaecologists, 2006.

2 Odd DE, Lewis G, Whitelaw A, Gunnell D. Resuscitation at birth and cognition at 8 years of age: a cohort study. Lancet 2009;373:1615-22.

3 Mercer J, Bewley S. Could early cord clamping harm neonatal stabilisation? Lancet 2009;374:377-8.

\section{In response}

Our comments were meant to indicate that cord blood stem cells, as compared to embryonic stem cells, present 'little ethical challenge' (particularly in the USA). Cord blood is readily available with more than four million births per year in the USA; many more than there are monies available for collection in both public and private banks. Therefore, in the USA it is common practice to not alter in any fashion the standard birthing procedures for the collector. If for some reason, inadequate volumes are collected the samples may be discarded. We firmly agree that the health and well being of the infant and mother are always of primary importance. Further, as was noted in our article, cord blood stem cell therapies have shown promise for treatment of adult as well as neonate disease such as cerebral palsy. ${ }^{1}$

Having said that many professionals argue that early clamping (within the first 30 seconds of birth) allows for more immediate care of the newborn and mother while others argue that late clamping (after two minutes or more) may allow for increased transfer of blood from the placenta to the newborn. There is evidence to support both sides of this argument, derived from studies conducted in North America, England, Australia and South America, including increased deleterious haemoglobin content, increased incidence of jaundice and polycythemia in infants delivered using late clamping. ${ }^{2-5}$ However, early clamping has been thought to possibly deprive the newborn of potentially needed red cells and iron. ${ }^{2-5}$

Regardless, in the USA it has been common practice for more than 50 years to deliver infants utilising what would be 